\title{
In vitro Compatibility between Botanicals and Pesticides against Xanthomonas axonopodis pv. punicae
}

\author{
Jagadeesh Bagewadi*, K.C. Kirankumar, R.K. Mestha and K. Chetankumar \\ Department of Plant pathology, University of Horticultural Sciences, Bagalkot, \\ Karnataka, India \\ *Corresponding author
}

\begin{abstract}
A B S T R A C T
Keywords

Botanicals,

Pesticides and

Compatibility

Article Info

Accepted:

16 February 2018

Available Online:

10 March 2018

The study reveals the possibility to use simultaneously botanicals and pesticide control products as efficient and environmental friendly for disease control, which could provide a decrease of the pesticide dose needed for plant protection. This experiment reports the in vitro compatibility of botanicals viz., Garcinia indica and Prosopis juliflora and pesticides Viz., Streptocycline, 2-bromo-2-nitropropane-1,3-diol,Carbendazim, Difenconazole, Hexaconazole, Propiconazol, Chlorothalonil, Copper oxychloride, Mancozeb, Chlorpyrifos, Dichlorovas and Dimethoate. Results indicate that, among the pesticides, 2bromo-2-nitropropane-1,3-diol(bronopol) in combination with Prosopi sjuliflora and Garcinia indica recorded highest average zone of inhibition of 32.11 and $31.11 \mathrm{~mm}$. Followed by Prosopis juliflora@ @1:5+Streptocycline 500ppm (30.78 mm) and Garcinia indica@1:5+Streptocycline 500ppm (28.66 mm).
\end{abstract}

\section{Introduction}

Bacterial blight caused by Xanthomonas axonopodis pv. punicae is a major concern. Since then several workers have reported the occurrence of blight and resultant losses from different states of the country viz., Tamil Nadu (Rangaswami, 1962), Himachal Pradesh (Sohi et al., 1964), Haryana (Kanwar, 1976), Karnataka (Chand and Kishun, 1991), Maharashtra (Kamble, 1990) and Punjab (Rani and Verma, 2001). Stem infection is also observed in severely infected orchards with girdling and cracking symptoms. Spots on fruits are variably dark brown in colour, irregular, slightly raised with oily appearance, which splits open with L or Y-shaped cracks under severe stages of disease (Bora and Kataki, 2014). Use of chemicals is not only the solution for management of any disease, while its use leads to pollution and hazard to the ecosystem.

Apart from the fact that pomegranate is largely exported to European and American markets, they have rigid grading standards vice-versa., residual toxicity level for various pesticides, hence, the use of antibiotics/ bactericides is discouraged. Thus, there is a need to develop alternative and eco-friendly measures for controlling the disease. Greater emphasis has been placed towards development of biological pesticides, which includes botanicals. 


\section{Materials and Methods}

All the botanicals and their combinations were tested under laboratory condition by agar well diffusion method to know the compatibility between botanicals and pesticides. Compatibility of botanicals with different bactericides, fungicides and insecticides commonly used in pomegranate were tested under laboratory condition by agar well diffusion method (Table 1).

The compatibility of these botanicals was confirmed by Agar well diffusion method (Holder and Boyse, 1994). The culture of $X$. axonopodis pv. punicae was added to nutrient medium and poured in to the Petri plate and allowed solidify. The agar plates were punched aseptically with 6-mm sterilized cork borer to prepare wells. The wells were filled with $100 \mu \mathrm{l}$ of botanical extracts. All plates were incubated $\left(30^{\circ} \mathrm{C}\right)$ for $48 \mathrm{~h}$. After incubation, the diameters of any clear zones around the wells were measured in milli meter. Sterile water filled in agar well considered as negative control.

\section{Results and Discussion}

The results of the efficiency of $P$. juliflora in combination with different bactericides, contact and systemic fungicides and insecticides under in vitro were recorded and presented in Table 2. The pesticides were used in three different concentrations. Among the botanicals with bactericides, $P$. juliflora (1:5) with 2-bromo-2-nitropropane-1,3-diol (bronopol) found more efficient with the highest inhibition zones in diameter (28.67, 32.00 and $35.67 \mathrm{~mm}$ at 250,500 and $750 \mathrm{ppm}$ respectively). Streptocycline at 250, 500 and 750 ppm along with $P$. juliflora (1:5) recorded $27.67,30.00$ and $34.67 \mathrm{~mm}$ respectively. However 2-bromo-2-nitropropane-1,3-diol (bronopol) and Streptocycline alone recorded 24.67 and $22.00 \mathrm{~mm}$ at $250 \mathrm{ppm}, 25.33$ and
$24.00 \mathrm{~mm}$ at $500 \mathrm{ppm}$ and 28.67 and 26.67 $\mathrm{mm}$ at $750 \mathrm{ppm}$ respectively.

In case of systemic fungicide tested all the four systemic fungicides compatible with $P$. juliflora at their three different concentrations tested. Among these propiconazole 25\% EC at $0.5,1$ and $2 \mathrm{ml} /$ lit along with $P$. juliflora (1:5) recorded highest inhibition zone of 24.00, 26.00 and $30.67 \mathrm{~mm}$ respectively. This treatment followed by $P$. juliflora + hexaconazole (21.33, 22.67 and $25.33 \mathrm{~mm}), P$. juliflora $(1: 5)+$ difenconazole $(20.33,20.67$ and $26.00 \mathrm{~mm}$ ) and P. juliflora (1:5) + carbendazim (20.00, 22.67 and $25.00 \mathrm{~mm}$ at 1 , 2 and $3 \mathrm{~g} /$ lit respectively). However the propiconazole $25 \%$ EC (11.33, 12.67 and $15.33 \mathrm{~mm})$, hexaconazole $5 \mathrm{EC}(9.67,12.00$ and $14.33 \mathrm{~mm}$ ), difenconazole $(10.67,12.33$ and $14.67 \mathrm{~mm}$ ) with zone of inhibition in diameter at $0.5,1$ and $2 \mathrm{ml} /$ lit respectively and carbendazim 50 WP also recorded no inhibition zone at their three concentrations.

In case of contact fungicides tested all the three fungicides compatible with $P$. juliflora (1:5) at their three different concentrations tested. Among them copper oxychloride 50 $\mathrm{WP}$ at 1,2 and $3 \mathrm{~g} /$ lit along with $P$. juliflora (1:5) showed highest inhibition zone of 23.33, 24.00 and $26.00 \mathrm{~mm}$ respectively. Followed by $P$. juliflora (1:5) + mancozeb 75 WP (22.67, 24.00 and $25.33 \mathrm{~mm})$, P. juliflora (1:5) + chlorothalonil 75 WP (22.00, 23.33 and $24.33 \mathrm{~mm})$ at 1,2 and $3 \mathrm{~g} /$ lit respectively. Whereas copper oxychloride 50 WP (14.67, 21.33 and $23.33 \mathrm{~mm}$ ), mancozeb $75 \mathrm{WP}$ $(11.33, \quad 15.67$ and $24.00 \mathrm{~mm})$ and chlorothalonil 75 WP (8.76, 14.00 and 17.67 $\mathrm{mm}$ ) alone recorded zone of inhibition in diameter at 1,2 and $3 \mathrm{~g} /$ lit respectively.

However, in case of insecticides, all three insecticides are compatible with $P$. juliflora (1:5) at their three different concentrations tested. 
Table.1 Treatment used in compatibility of botanicals with commonly using pesticides in pomegranate

\begin{tabular}{|c|c|c|c|c|c|}
\hline Treat & Chemicals & & \multicolumn{3}{|c|}{ Concentration } \\
\hline \multicolumn{3}{|c|}{ Bactericides } & $\mathbf{L}$ & $\mathbf{M}$ & H \\
\hline$\overline{T_{1}}$ & $\begin{array}{l}\text { 2-bromo-2-nitropropane- } \\
\text { 1,3-diol(bronopol) }\end{array}$ & $\begin{array}{l}\text { Multiplex agricareP } \\
\text { Ltd }\end{array}$ & $250 \mathrm{ppm}$ & $\begin{array}{l}500 \\
\mathrm{ppm}\end{array}$ & $750 \mathrm{ppm}$ \\
\hline $\mathbf{T}_{2}$ & K-cyclin & - & $250 \mathrm{ppm}$ & $\begin{array}{r}500 \\
\mathrm{ppm}\end{array}$ & $750 \mathrm{ppm}$ \\
\hline $\mathbf{T}_{3}$ & $\begin{array}{l}\text { Streptomycin } \\
\text { sulphate90\%+Tetracyclin } \\
\text { e } \\
\text { hydrochloride+10\%(Stre } \\
\text { ptocyclin) }\end{array}$ & $\begin{array}{l}\text { Hindustan } \\
\text { Antibiotics Ltd, Pune }\end{array}$ & 100 ppm & $\begin{array}{l}250 \\
\mathrm{ppm}\end{array}$ & $500 \mathrm{ppm}$ \\
\hline \multicolumn{6}{|c|}{ Fungicides } \\
\hline $\mathbf{T}_{4}$ & $\begin{array}{l}\text { Carbendazim } 50 \mathrm{WP} \\
\text { (Bavistin) }\end{array}$ & $\begin{array}{l}\text { BASF, West } \\
\text { Germany }\end{array}$ & 1g/lit & $2 \mathrm{~g} / \mathrm{lit}$ & $2.5 \mathrm{~g} / \mathrm{lit}$ \\
\hline$T_{5}$ & $\begin{array}{l}\text { Chlorothalonil } 75 \\
\text { WP(Kavach) }\end{array}$ & SDS Biotech. KK & $1.0 \mathrm{~g} / \mathrm{lit}$ & $2.0 \mathrm{~g} / \mathrm{lit}$ & $3.0 \mathrm{~g} / \mathrm{lit}$ \\
\hline$T_{6}$ & $\begin{array}{l}\text { Copper oxychloride } 50 \\
\text { WP (Blitox) }\end{array}$ & $\begin{array}{l}\text { Artee Graphite P } \\
\text { Ltd., Delhi. }\end{array}$ & 1g/lit & $2.0 \mathrm{~g} / \mathrm{lit}$ & $3 \mathrm{~g} / \mathrm{lit}$ \\
\hline $\mathbf{T}_{7}$ & $\begin{array}{l}\text { Difenconazole } 25 \text { EC } \\
\text { (Score) }\end{array}$ & $\begin{array}{l}\text { Syngenta Crop } \\
\text { Protection AG } \\
\text { Monthey, } \\
\text { Switzerland }\end{array}$ & $0.5 \mathrm{ml} / \mathrm{lit}$ & $1 \mathrm{ml} / \mathrm{lit}$ & $2 \mathrm{ml} / \mathrm{lit}$ \\
\hline$T_{8}$ & $\begin{array}{l}\text { Hexaconozole } 5 \text { EC } \\
\text { (Contaf) }\end{array}$ & Rallis India Ltd. & $0.5 \mathrm{ml} / \mathrm{lit}$ & $1 \mathrm{ml} / \mathrm{lit}$ & $1.5 \mathrm{ml} / \mathrm{lit}$ \\
\hline$T_{9}$ & $\begin{array}{l}\text { Mancozeb75 WP (Indofil } \\
\text { M-45) }\end{array}$ & $\begin{array}{l}\text { Dow Agro Science, } \\
\text { USA }\end{array}$ & $1 \mathrm{~g} / \mathrm{lit}$ & $2-3 \mathrm{~g} / \mathrm{ml}$ & $4 \mathrm{~g} / \mathrm{lit}$ \\
\hline$T_{10}$ & $\begin{array}{l}\text { Propiconazole 25\% EC } \\
\text { (Tilt) }\end{array}$ & $\begin{array}{l}\text { Syngenta Crop } \\
\text { Protection AG., } \\
\text { Monthey, } \\
\text { Switzerland }\end{array}$ & $0.5 \mathrm{ml} / \mathrm{lit}$ & $1 \mathrm{ml} / \mathrm{lit}$ & $2 \mathrm{ml} /$ lit \\
\hline \multicolumn{6}{|c|}{ Insecticides } \\
\hline $\mathrm{T}_{11}$ & $\begin{array}{l}\text { Chlorpyrifos 20\% EC } \\
\text { (Predator) }\end{array}$ & $\begin{array}{l}\text { Dow Agro Sciences } \\
\text { LLC, USA }\end{array}$ & $1.5 \mathrm{ml} / \mathrm{lit}$ & $2 \mathrm{ml} / \mathrm{lit}$ & $2.5 \mathrm{ml} / / \mathrm{lit}$ \\
\hline$T_{12}$ & $\begin{array}{l}\text { Dichlorovas } 76 \% \text { EC } \\
\text { (Nuvan) }\end{array}$ & $\begin{array}{l}\text { Makhteshim Agan, } \\
\text { Israel. }\end{array}$ & $0.25 \%$ & $0.5 \%$ & $0.1 \%$ \\
\hline$T_{13}$ & $\begin{array}{l}\text { Dimethoate 30\% EC } \\
\text { (Rogor) }\end{array}$ & Agrimot SPA, Italy & $0.5 \mathrm{ml} / \mathrm{lit}$ & $1 \mathrm{~m} / 1 \mathrm{lit}$ & 2ml/lit \\
\hline $\mathrm{T}_{14}$ & $\begin{array}{l}\text { Imidachloprid 70\%WG } \\
\text { (Admire) }\end{array}$ & $\begin{array}{l}\text { Bayer Crop Science, } \\
\text { AG, Germany }\end{array}$ & $0.15 \mathrm{ml} / \mathrm{lit}$ & $0.3 \mathrm{ml} / \mathrm{lit}$ & $0.45 \mathrm{ml} / \mathrm{lit}$ \\
\hline$T_{15}$ & Control & - & - & - & - \\
\hline
\end{tabular}


Table. 2 Compatibility of Prosopis juliflora with pesticides against Xanthomonas axonopodis pv. punicae under in vitro condition

\begin{tabular}{|c|c|c|c|c|c|}
\hline Treat ments & Treatment details & \multicolumn{4}{|c|}{ Zone of inhibition (mean diameter in $\mathrm{mm}$ ) } \\
\hline $\mathbf{T}_{1}$ & Prosopisjuliflora(1:5) & \multicolumn{4}{|c|}{$22.33(4.83)$} \\
\hline \multicolumn{2}{|r|}{ Bactericides } & $\mathbf{L}^{*}$ & $\mathbf{M}^{*}$ & $\mathbf{H}^{*}$ & Average \\
\hline $\mathbf{T}_{2}$ & Prosopisjuliflora+ Streptocycline (L= 250 ppm, M=500ppm, H=750ppm) & $\begin{array}{l}27.67 \\
(5.26)\end{array}$ & $\begin{array}{l}30.00 \\
(5.57)\end{array}$ & $\begin{array}{l}34.67 \\
(5.97)\end{array}$ & $\begin{array}{l}30.78 \\
(5.60)\end{array}$ \\
\hline $\mathbf{T}_{3}$ & $\begin{array}{l}\text { Prosopis juliflora + 2-bromo-2-nitropropane-1,3-diol ( } \mathrm{L}=0.25 \mathrm{ppm}, \mathrm{M}=0.5 \mathrm{ppm} \text {, } \\
\mathrm{H}=0.75 \mathrm{ppm})\end{array}$ & $\begin{array}{l}28.67 \\
(5.45)\end{array}$ & $\begin{array}{l}32.00 \\
(5.74)\end{array}$ & $\begin{array}{l}35.67 \\
(6.05)\end{array}$ & $\begin{array}{l}32.11 \\
(5.75)\end{array}$ \\
\hline $\mathbf{T}_{4}$ & Streptocycline $(\mathrm{L}=250 \mathrm{ppm}, \mathrm{M}=500 \mathrm{ppm}, \mathrm{H}=750 \mathrm{ppm})$ & $\begin{array}{l}22.00 \\
(4.79)\end{array}$ & $\begin{array}{l}24.00 \\
(5.01)\end{array}$ & $\begin{array}{l}26.67 \\
(5.26)\end{array}$ & $\begin{array}{l}24.22 \\
(5.17)\end{array}$ \\
\hline $\mathbf{T}_{5}$ & 2-bromo-2-nitropropane-1,3-diol ( $\mathrm{L}=0.25 \mathrm{ppm}, \mathrm{M}=0.5 \mathrm{ppm}, \mathrm{H}=0.75 \mathrm{ppm})$ & $\begin{array}{l}24.67 \\
(5.07)\end{array}$ & $\begin{array}{l}25.33 \\
(5.13)\end{array}$ & $\begin{array}{l}28.67 \\
(5.45)\end{array}$ & $\begin{array}{l}26.20 \\
(5.01)\end{array}$ \\
\hline \multicolumn{6}{|c|}{ Systemic fungicides } \\
\hline$T_{6}$ & Prosopisjuliflora + Carbendazim $50 \mathrm{WP}(\mathrm{L}=1 \mathrm{~g} / \mathrm{lit}, \mathrm{M}=2 \mathrm{~g} / \mathrm{lit}, \mathrm{H}=3 \mathrm{~g} / \mathrm{lit})$ & $\begin{array}{l}20.00 \\
(4.58)\end{array}$ & $\begin{array}{l}22.67 \\
(4.86)\end{array}$ & $\begin{array}{l}25.00 \\
(5.01)\end{array}$ & $\begin{array}{l}22.55 \\
(4.85)\end{array}$ \\
\hline $\mathbf{T}_{7}$ & Prosopisjuliflora + Difenconazole $25 \mathrm{EC}(\mathrm{L}=0.5 \mathrm{ml} / \mathrm{lit}, \mathrm{M}=1 \mathrm{ml} / \mathrm{lit}, \mathrm{H}=2 \mathrm{ml} / \mathrm{lit})$ & $\begin{array}{l}20.33 \\
(4.62)\end{array}$ & $\begin{array}{l}20.67 \\
(4.65)\end{array}$ & $\begin{array}{l}26.00 \\
(5.19)\end{array}$ & $\begin{array}{l}22.33 \\
(4.82)\end{array}$ \\
\hline $\mathbf{T}_{8}$ & Prosopisjuliflora + Hexaconozole $5 \mathrm{EC} \mathrm{(L=0.5} \mathrm{ml/lit,} \mathrm{M=1ml/lit,} \mathrm{H}=2 \mathrm{ml} / \mathrm{lit})$ & $\begin{array}{l}21.33 \\
(4.72)\end{array}$ & $\begin{array}{l}22.67 \\
(4.86)\end{array}$ & $\begin{array}{l}25.33 \\
(5.13)\end{array}$ & $\begin{array}{l}23.11 \\
(4.91)\end{array}$ \\
\hline $\mathbf{T}_{9}$ & Prosopisjuliflora + Propiconazole 25EC (L= $0.5 \mathrm{ml} / \mathrm{lit}, \mathrm{M}=1 \mathrm{ml} / \mathrm{lit}, \mathrm{H}=2 \mathrm{ml} / \mathrm{lit})$ & $\begin{array}{l}24.00 \\
(4.89)\end{array}$ & $\begin{array}{l}26.00 \\
(5.20)\end{array}$ & $\begin{array}{l}30.67 \\
(5.63)\end{array}$ & $\begin{array}{l}26.89 \\
(5.27)\end{array}$ \\
\hline $\mathbf{T}_{10}$ & Carbendazim 50 WP $(\mathrm{L}=1 \mathrm{~g} / \mathrm{lit}, \mathrm{M}=2 \mathrm{~g} / \mathrm{lit}, \mathrm{H}=3 \mathrm{~g} / \mathrm{lit})$ & $\begin{array}{c}0.00 \\
(1.41)\end{array}$ & $\begin{array}{c}0.00 \\
(1.41)\end{array}$ & $\begin{array}{c}0.00 \\
(1.41)\end{array}$ & $\begin{array}{c}0.00 \\
(1.41)\end{array}$ \\
\hline $\mathbf{T}_{11}$ & Difenconazole $25 \mathrm{EC}(\mathrm{L}=0.5 \mathrm{ml} / \mathrm{lit}, \mathrm{M}=1 \mathrm{ml} / \mathrm{lit}, \mathrm{H}=2 \mathrm{ml} / \mathrm{lit})$ & $\begin{array}{l}10.67 \\
(3.41)\end{array}$ & $\begin{array}{l}12.33 \\
(3.65)\end{array}$ & $\begin{array}{l}14.67 \\
(3.96)\end{array}$ & $\begin{array}{l}12.55 \\
(3.67)\end{array}$ \\
\hline $\mathbf{T}_{12}$ & Hexaconozole $5 \mathrm{EC}(\mathrm{L}=0.5 \mathrm{ml} / \mathrm{lit}, \mathrm{M}=1 \mathrm{ml} / \mathrm{lit}, \mathrm{H}=2 \mathrm{ml} / \mathrm{lit})$ & $\begin{array}{c}9.67 \\
(3.26)\end{array}$ & $\begin{array}{l}12.00 \\
(3.60)\end{array}$ & $\begin{array}{c}14.33 \\
(3.92)\end{array}$ & $\begin{array}{l}12.00 \\
(3.59)\end{array}$ \\
\hline $\mathbf{T}_{13}$ & Propiconazole $25 \% \mathrm{EC}(\mathrm{L}=0.5 \mathrm{ml} / \mathrm{lit}, \mathrm{M}=1 \mathrm{ml} / \mathrm{lit}, \mathrm{H}=2 \mathrm{ml} / \mathrm{lit})$ & $\begin{array}{l}11.33 \\
(3.51)\end{array}$ & $\begin{array}{l}12.67 \\
(3.70)\end{array}$ & $\begin{array}{c}15.33 \\
(3.04)\end{array}$ & $\begin{array}{l}13.11 \\
(3.75)\end{array}$ \\
\hline \multicolumn{6}{|c|}{ Contact fungicides } \\
\hline $\mathbf{T}_{14}$ & Prosopisjuliflora + Chlorothalonil 75WP $(\mathrm{L}=1 \mathrm{~g} / \mathrm{lit}, \mathrm{M}=2 \mathrm{~g} / \mathrm{lit}, \mathrm{H}=3 \mathrm{~g} / \mathrm{lit})$ & $\begin{array}{l}22.00 \\
(4.79)\end{array}$ & $\begin{array}{l}23.33 \\
(4.93)\end{array}$ & $\begin{array}{l}24.33 \\
(4.93)\end{array}$ & $\begin{array}{l}23.22 \\
(5.01)\end{array}$ \\
\hline $\mathbf{T}_{15}$ & Prosopisjuliflora + Copper oxychloride $550 \mathrm{WP}(\mathrm{L}=1 \mathrm{~g} / \mathrm{lit}, \mathrm{M}=2 \mathrm{~g} / \mathrm{lit}, \mathrm{H}=3 \mathrm{~g} / \mathrm{lit})$ & $\begin{array}{l}23.33 \\
(4.93)\end{array}$ & $\begin{array}{l}24.00 \\
(4.89)\end{array}$ & $\begin{array}{l}26.00 \\
(5.12)\end{array}$ & $\begin{array}{l}24.44 \\
(5.04)\end{array}$ \\
\hline $\mathbf{T}_{16}$ & Prosopisjuliflora + Mancozeb75 WP (L=1g/lit, M=2g/lit, H=3g/lit) & $\begin{array}{l}22.67 \\
(4.86)\end{array}$ & $\begin{array}{l}24.00 \\
(4.89)\end{array}$ & $\begin{array}{l}25.33 \\
(5.13)\end{array}$ & $\begin{array}{l}24.00 \\
(5.01)\end{array}$ \\
\hline $\mathbf{T}_{17}$ & Chlorothalonil 75WP $(\mathrm{L}=1 \mathrm{~g} / \mathrm{lit}, \mathrm{M}=2 \mathrm{~g} / \mathrm{lit}, \mathrm{H}=3 \mathrm{~g} / \mathrm{lit})$ & $\begin{array}{c}8.67 \\
(3.11)\end{array}$ & $\begin{array}{l}14.00 \\
(3.87)\end{array}$ & $\begin{array}{l}17.67 \\
(4.32)\end{array}$ & $\begin{array}{l}13.44 \\
(3.77)\end{array}$ \\
\hline $\mathbf{T}_{18}$ & Copper oxychloride $550 \mathrm{WP}(\mathrm{L}=1 \mathrm{~g} / \mathrm{lit}, \mathrm{M}=2 \mathrm{~g} / \mathrm{lit}, \mathrm{H}=3 \mathrm{~g} / \mathrm{lit})$ & $\begin{array}{l}14.67 \\
(3.96)\end{array}$ & $\begin{array}{l}21.33 \\
(4.73)\end{array}$ & $\begin{array}{l}23.33 \\
(4.93)\end{array}$ & $\begin{array}{l}19.77 \\
(4.54)\end{array}$ \\
\hline $\mathbf{T}_{19}$ & Mancozeb75 WP (L=1g/lit, M=2g/lit, H=3g/lit) & $\begin{array}{l}11.33 \\
(3.51)\end{array}$ & $\begin{array}{l}15.67 \\
(3.96)\end{array}$ & $\begin{array}{l}24.00 \\
(4.51)\end{array}$ & $\begin{array}{l}17.00 \\
(4.00)\end{array}$ \\
\hline \multicolumn{6}{|c|}{ Insecticides } \\
\hline $\mathbf{T}_{20}$ & Prosopisjuliflora + Chlorpyrifos $20 \% \mathrm{EC}(\mathrm{L}=1.5 \mathrm{ml} / \mathrm{lit}, \mathrm{M}=2 \mathrm{ml} / \mathrm{lit}, \mathrm{H}=3 \mathrm{ml} / \mathrm{lit})$ & $\begin{array}{l}22.00 \\
(4.79)\end{array}$ & $\begin{array}{l}22.33 \\
(4.83)\end{array}$ & $\begin{array}{l}26.67 \\
(5.26)\end{array}$ & $\begin{array}{l}23.66 \\
(4.96)\end{array}$ \\
\hline $\mathbf{T}_{21}$ & Prosopis juliflora + Dichlorovas $76 \% \mathrm{EC}(\mathrm{L}=0.25 \%, \mathrm{M}=0.5 \%, \mathrm{H}=1.0 \%)$ & $\begin{array}{l}20.00 \\
(4.58)\end{array}$ & $\begin{array}{l}22.33 \\
(4.83)\end{array}$ & $\begin{array}{l}25.33 \\
(5.13)\end{array}$ & $\begin{array}{l}22.55 \\
(4.85)\end{array}$ \\
\hline $\mathbf{T}_{22}$ & Prosopisjuliflora + Dimethoate $30 \% \mathrm{EC}(\mathrm{L}=0.5 \mathrm{ml} / \mathrm{lit}, \mathrm{M}=1 \mathrm{ml} / \mathrm{lit}, \mathrm{H}=2 \mathrm{ml} / \mathrm{lit})$ & $\begin{array}{l}22.67 \\
(4.86)\end{array}$ & $\begin{array}{l}23.67 \\
(4.97)\end{array}$ & $\begin{array}{l}26.67 \\
(5.26)\end{array}$ & $\begin{array}{l}23.89 \\
(5.02)\end{array}$ \\
\hline $\mathbf{T}_{23}$ & Chlorpyrifos 20\% EC (L=1.5 ml/lit, M=2ml/lit, $\mathrm{H}=3 \mathrm{ml} / \mathrm{lit})$ & $\begin{array}{c}0.00 \\
(1.41)\end{array}$ & $\begin{array}{c}0.00 \\
(1.41)\end{array}$ & $\begin{array}{c}0.00 \\
(1.41)\end{array}$ & $\begin{array}{c}0.00 \\
(1.41)\end{array}$ \\
\hline $\mathbf{T}_{24}$ & Dichlorovas $76 \% \mathrm{EC}(\mathrm{L}=0.25 \%, \mathrm{M}=0.5 \%, \mathrm{H}=1.0 \%)$ & $\begin{array}{c}0.00 \\
(1.41)\end{array}$ & $\begin{array}{c}0.00 \\
(1.41)\end{array}$ & $\begin{array}{c}0.00 \\
(1.41)\end{array}$ & $\begin{array}{c}0.00 \\
(1.41)\end{array}$ \\
\hline $\mathbf{T}_{25}$ & Dimethoate $30 \% \mathrm{EC} \mathrm{(L=0.5} \mathrm{ml/lit,} \mathrm{M}=1 \mathrm{ml} / \mathrm{lit}, \mathrm{H}=2 \mathrm{ml} / \mathrm{lit})$ & $\begin{array}{c}0.00 \\
(1.41)\end{array}$ & $\begin{array}{c}0.00 \\
(1.41)\end{array}$ & $\begin{array}{c}0.00 \\
(1.41)\end{array}$ & $\begin{array}{c}0.00 \\
(1.41)\end{array}$ \\
\hline \multirow[t]{2}{*}{$\mathbf{T}_{26}$} & Control & $\begin{array}{c}0.00 \\
(1.41)\end{array}$ & $\begin{array}{c}0.00 \\
(1.41)\end{array}$ & $\begin{array}{c}0.00 \\
(1.41)\end{array}$ & $\begin{array}{c}0.00 \\
(1.41)\end{array}$ \\
\hline & Between treatments & \multicolumn{2}{|c|}{ Between dilutions } & \multicolumn{2}{|c|}{ Interactions } \\
\hline SEm \pm & 0.04 & \multicolumn{2}{|c|}{0.01} & \multicolumn{2}{|c|}{0.07} \\
\hline CD (0.01) & 0.12 & \multicolumn{2}{|c|}{0.04} & \multicolumn{2}{|c|}{0.20} \\
\hline
\end{tabular}

*The results in the parentheses are square root transformed values

Note*: $^{*} \mathrm{~L}=$ Lower concentration, $\mathrm{M}=$ Medium concentration, $\mathrm{H}=$ Higher concentration 
Table.3 Compatibility of Garcinia indica with pesticides against Xanthomonas axonopodis pv. punicae under in vitro condition

\begin{tabular}{|c|c|c|c|c|c|}
\hline Treatments & Treatment details & \multicolumn{4}{|c|}{ Zone of inhibition (mean diameter in mm) } \\
\hline $\mathbf{T}_{1}$ & Garciniaindica(1:5) & \multicolumn{4}{|c|}{$20.44(4.52)$} \\
\hline \multicolumn{2}{|r|}{ Bactericides } & $\mathbf{L}^{*}$ & $\mathbf{M}^{*}$ & $\mathbf{H}^{*}$ & Average \\
\hline $\mathbf{T}_{2}$ & Garcinia indica + Streptocycline $(\mathrm{L}=250 \mathrm{ppm}, \mathrm{M}=500 \mathrm{ppm}, \mathrm{H}=750 \mathrm{ppm})$ & $\begin{array}{l}25.33 \\
(5.13)\end{array}$ & $\begin{array}{l}28.00 \\
(5.39)\end{array}$ & $\begin{array}{l}32.67 \\
(5.80)\end{array}$ & $\begin{array}{l}28.66 \\
(4.44)\end{array}$ \\
\hline $\mathbf{T}_{3}$ & $\begin{array}{l}\text { Garcinia indica }+2 \text {-bromo-2-nitropropane-1,3-diol (L= } 250 \mathrm{ppm}, \mathrm{M}=500 \mathrm{ppm} \text {, } \\
\mathrm{H}=750 \mathrm{ppm})\end{array}$ & $\begin{array}{l}27.33 \\
(5.32)\end{array}$ & $\begin{array}{l}32.00 \\
(5.57)\end{array}$ & $\begin{array}{l}34.00 \\
(5.92)\end{array}$ & $\begin{array}{l}31.11 \\
(5.60)\end{array}$ \\
\hline $\mathbf{T}_{4}$ & Streptocycline $(\mathrm{L}=250 \mathrm{ppm}, \mathrm{M}=500 \mathrm{ppm}, \mathrm{H}=750 \mathrm{ppm})$ & $\begin{array}{l}22.00 \\
(4.79)\end{array}$ & $\begin{array}{l}24.00 \\
(4.89)\end{array}$ & $\begin{array}{l}26.67 \\
(5.26)\end{array}$ & $\begin{array}{l}24.22 \\
(5.02)\end{array}$ \\
\hline $\mathbf{T}_{5}$ & 2-bromo-2-nitropropane-1,3-diol ( $\mathrm{L}=250 \mathrm{ppm}, \mathrm{M}=500 \mathrm{ppm}, \mathrm{H}=750 \mathrm{ppm})$ & $\begin{array}{l}24.67 \\
(5.07)\end{array}$ & $\begin{array}{l}25.33 \\
(5.13)\end{array}$ & $\begin{array}{l}28.67 \\
(5.45)\end{array}$ & $\begin{array}{l}26.22 \\
(5.21)\end{array}$ \\
\hline \multicolumn{6}{|c|}{ Systemic fungicides } \\
\hline $\mathbf{T}_{6}$ & Garciniaindica + Carbendazim $50 \mathrm{WP}(\mathrm{L}=1 \mathrm{~g} / \mathrm{lit}, \mathrm{M}=2 \mathrm{~g} / \mathrm{lit}, \mathrm{H}=3 \mathrm{~g} / \mathrm{lit})$ & $\begin{array}{l}17.00 \\
(4.24)\end{array}$ & $\begin{array}{l}20.00 \\
(4.58)\end{array}$ & $\begin{array}{l}21.00 \\
(4.70)\end{array}$ & $\begin{array}{l}19.33 \\
(4.51)\end{array}$ \\
\hline $\mathbf{T}_{7}$ & Garcinia indica + Difenconazole $25 \mathrm{EC} \mathrm{(L=0.5} \mathrm{ml/lit,} \mathrm{M=1ml/lit,} \mathrm{H=2ml/lit)}$ & $\begin{array}{l}18.00 \\
(4.36)\end{array}$ & $\begin{array}{l}20.33 \\
(4.62)\end{array}$ & $\begin{array}{l}22.67 \\
(4.87)\end{array}$ & $\begin{array}{l}20.33 \\
(4.61)\end{array}$ \\
\hline$T_{8}$ & Garcinia indica + Hexaconozole $5 \mathrm{EC}(\mathrm{L}=0.5 \mathrm{ml} / \mathrm{lit}, \mathrm{M}=1 \mathrm{ml} / \mathrm{lit}, \mathrm{H}=2 \mathrm{ml} / \mathrm{lit})$ & $\begin{array}{l}20.00 \\
(4.58)\end{array}$ & $\begin{array}{l}23.33 \\
(4.93)\end{array}$ & $\begin{array}{l}25.00 \\
(5.10)\end{array}$ & $\begin{array}{l}22.77 \\
(4.87)\end{array}$ \\
\hline$T_{9}$ & Garcinia indica + Propiconazole $25 \mathrm{EC} \mathrm{(L=0.5} \mathrm{ml/lit,} \mathrm{M=1ml/lit,} \mathrm{H=2ml/lit)}$ & $\begin{array}{l}22.00 \\
(4.79)\end{array}$ & $\begin{array}{l}24.00 \\
(4.89)\end{array}$ & $\begin{array}{l}26.00 \\
(5.20)\end{array}$ & $\begin{array}{l}24.00 \\
(5.00)\end{array}$ \\
\hline$T_{10}$ & Carbendazim $50 \mathrm{WP}(\mathrm{L}=1 \mathrm{~g} / \mathrm{lit}, \mathrm{M}=2 \mathrm{~g} / \mathrm{lit}, \mathrm{H}=3 \mathrm{~g} / \mathrm{lit})$ & $\begin{array}{c}0.00 \\
(1.41)\end{array}$ & $\begin{array}{c}0.00 \\
(1.41)\end{array}$ & $\begin{array}{c}0.00 \\
(1.41)\end{array}$ & $\begin{array}{c}0.00 \\
(1.41)\end{array}$ \\
\hline $\mathbf{T}_{11}$ & Difenconazole $25 \mathrm{EC}(\mathrm{L}=0.5 \mathrm{ml} / \mathrm{lit}, \mathrm{M}=1 \mathrm{ml} / \mathrm{lit}, \mathrm{H}=2 \mathrm{ml} / \mathrm{lit})$ & $\begin{array}{l}10.67 \\
(3.41)\end{array}$ & $\begin{array}{l}12.33 \\
(3.65)\end{array}$ & $\begin{array}{l}13.33 \\
(3.96)\end{array}$ & $\begin{array}{l}12.11 \\
(3.67)\end{array}$ \\
\hline$T_{12}$ & 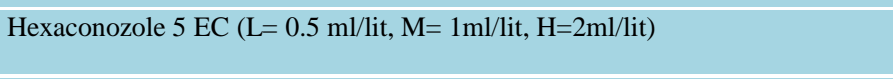 & $\begin{array}{c}9.67 \\
(3.26)\end{array}$ & $\begin{array}{l}12.00 \\
(3.60)\end{array}$ & $\begin{array}{l}13.33 \\
(3.92)\end{array}$ & $\begin{array}{l}11.66 \\
(3.60)\end{array}$ \\
\hline $\mathbf{T}_{13}$ & Propiconazole $25 \mathrm{EC}(\mathrm{L}=0.5 \mathrm{ml} / \mathrm{lit}, \mathrm{M}=1 \mathrm{ml} / \mathrm{lit}, \mathrm{H}=2 \mathrm{ml} / \mathrm{lit})$ & $\begin{array}{l}11.33 \\
(3.51)\end{array}$ & $\begin{array}{c}12.67 \\
(3.700)\end{array}$ & $\begin{array}{l}13.67 \\
(4.04)\end{array}$ & $\begin{array}{l}12.55 \\
(3.75)\end{array}$ \\
\hline \multicolumn{6}{|c|}{ Contact fungicides } \\
\hline $\mathbf{T}_{14}$ & Garciniaindica + Chlorothalonil 75WP (L=1g/lit, M=2g/lit, H=3g/lit) & $\begin{array}{l}19.00 \\
(4.73)\end{array}$ & $\begin{array}{l}20.67 \\
(5.07)\end{array}$ & $\begin{array}{l}23.00 \\
(5.30)\end{array}$ & $\begin{array}{l}20.89 \\
(5.03)\end{array}$ \\
\hline $\mathbf{T}_{15}$ & Garciniaindica + Copper oxychloride 50\% WP (L=1g/lit, M=2g/lit, H=3g/lit) & $\begin{array}{l}21.33 \\
(4.65)\end{array}$ & $\begin{array}{l}24.67 \\
(4.23)\end{array}$ & $\begin{array}{l}27.00 \\
(4.90)\end{array}$ & $\begin{array}{l}24.33 \\
(4.93)\end{array}$ \\
\hline $\mathbf{T}_{16}$ & Garciniaindica + Mancozeb75 WP (L=1g/lit, M=2g/lit, H=3g/lit) & $\begin{array}{l}20.67 \\
(4.47)\end{array}$ & $\begin{array}{l}23.00 \\
(4.65)\end{array}$ & $\begin{array}{l}26.33 \\
(4.90)\end{array}$ & $\begin{array}{l}23.33 \\
(4.67)\end{array}$ \\
\hline $\mathbf{T}_{17}$ & Chlorothalonil 75WP ( $\mathrm{L}=1 \mathrm{~g} / \mathrm{lit}, \mathrm{M}=2 \mathrm{~g} / \mathrm{lit}, \mathrm{H}=3 \mathrm{~g} / \mathrm{lit})$ & $\begin{array}{c}8.67 \\
(3.11)\end{array}$ & $\begin{array}{l}14.00 \\
(3.87)\end{array}$ & $\begin{array}{l}17.67 \\
(4.32)\end{array}$ & $\begin{array}{l}13.44 \\
(3.77)\end{array}$ \\
\hline $\mathbf{T}_{18}$ & Copper oxychloride50\% WP (L=1g/lit, M=2g/lit, H=3g/lit) & $\begin{array}{l}14.67 \\
(3.96)\end{array}$ & $\begin{array}{l}21.33 \\
(4.73)\end{array}$ & $\begin{array}{l}23.33 \\
(4.93)\end{array}$ & $\begin{array}{l}19.77 \\
(4.54)\end{array}$ \\
\hline $\mathbf{T}_{19}$ & Mancozeb75 WP (L=1g/lit, M=2g/lit, H=3g/lit) & $\begin{array}{r}11.33 \\
(3.51)\end{array}$ & $\begin{array}{r}15.67 \\
(3.96)\end{array}$ & $\begin{array}{l}19.33 \\
(4.51)\end{array}$ & $\begin{array}{l}15.44 \\
(4.00)\end{array}$ \\
\hline \multicolumn{6}{|c|}{ Insecticides } \\
\hline $\mathbf{T}_{20}$ & Garcinia indica + Chlorpyrifos $20 \% \mathrm{EC}(\mathrm{L}=1.5 \mathrm{ml} / \mathrm{lit}, \mathrm{M}=2 \mathrm{ml} / \mathrm{lit}, \mathrm{H}=3 \mathrm{ml} / \mathrm{lit})$ & $\begin{array}{l}18.67 \\
(4.43)\end{array}$ & $\begin{array}{l}20.67 \\
(4.65)\end{array}$ & $\begin{array}{l}23.00 \\
(4.90)\end{array}$ & $\begin{array}{l}20.78 \\
(4.66)\end{array}$ \\
\hline $\mathbf{T}_{21}$ & Garcinia indica + Dichlorovas $76 \% \mathrm{EC}(\mathrm{L}=0.25 \%, \mathrm{M}=0.5 \%, \mathrm{H}=1.0 \%)$ & $\begin{array}{l}17.67 \\
(4.32)\end{array}$ & $\begin{array}{l}20.00 \\
(4.58)\end{array}$ & $\begin{array}{l}21.33 \\
(4.73)\end{array}$ & $\begin{array}{l}19.66 \\
(4.54)\end{array}$ \\
\hline $\mathbf{T}_{22}$ & Garciniaindica + Dimethoate $30 \% \mathrm{EC}(\mathrm{L}=0.5 \mathrm{ml} / \mathrm{lit}, \mathrm{M}=1 \mathrm{ml} / \mathrm{lit}, \mathrm{H}=2 \mathrm{ml} / \mathrm{lit})$ & $\begin{array}{l}19.67 \\
(4.54)\end{array}$ & $\begin{array}{l}21.33 \\
(4.73)\end{array}$ & $\begin{array}{l}24.67 \\
(5.07)\end{array}$ & $\begin{array}{l}21.89 \\
(4.78)\end{array}$ \\
\hline $\mathbf{T}_{23}$ & Chlorpyrifos $20 \%$ EC (L=1.5 ml/lit, M= 2ml/lit, H=3ml/lit) & $\begin{array}{c}0.00 \\
(1.41)\end{array}$ & $\begin{array}{c}0.00 \\
(1.41)\end{array}$ & $\begin{array}{c}0.00 \\
(1.41)\end{array}$ & $\begin{array}{c}0.00 \\
(1.41)\end{array}$ \\
\hline $\mathbf{T}_{24}$ & Dichlorovas $76 \%$ EC $(\mathrm{L}=0.25 \%, \mathrm{M}=0.5 \%, \mathrm{H}=1.0 \%)$ & $\begin{array}{c}0.00 \\
(1.41)\end{array}$ & $\begin{array}{c}0.00 \\
(1.41)\end{array}$ & $\begin{array}{c}0.00 \\
(1.41)\end{array}$ & $\begin{array}{c}0.00 \\
(1.41)\end{array}$ \\
\hline $\mathbf{T}_{25}$ & Dimethoate $30 \%$ EC (L= $0.5 \mathrm{ml} / \mathrm{lit}, \mathrm{M}=1 \mathrm{ml} / \mathrm{lit}, \mathrm{H}=2 \mathrm{ml} / \mathrm{lit})$ & $\begin{array}{c}0.00 \\
(1.41)\end{array}$ & $\begin{array}{c}0.00 \\
(1.41)\end{array}$ & $\begin{array}{c}0.00 \\
(1.41)\end{array}$ & $\begin{array}{c}0.00 \\
(1.41)\end{array}$ \\
\hline \multirow[t]{2}{*}{$\mathbf{T}_{26}$} & Control & $\begin{array}{c}0.00 \\
(1.41)\end{array}$ & $\begin{array}{c}0.00 \\
(1.41)\end{array}$ & $\begin{array}{c}0.00 \\
(1.41)\end{array}$ & $\begin{array}{c}0.00 \\
(1.41)\end{array}$ \\
\hline & Between treatments & \multicolumn{2}{|c|}{ Between dilutions } & \multicolumn{2}{|c|}{ Interaction } \\
\hline SEm \pm & 0.03 & \multicolumn{2}{|c|}{0.01} & \multicolumn{2}{|c|}{0.06} \\
\hline $\mathrm{CD}(0.01)$ & 0.10 & \multicolumn{2}{|c|}{0.03} & \multicolumn{2}{|c|}{0.18} \\
\hline
\end{tabular}

*The results in the parentheses are square root transformed values

Note*: L= Lower concentration, $\mathrm{M}=$ Medium concentration, $\mathrm{H}=\mathrm{Higher}$ concentration. 
Among them dimethoate $30 \% \mathrm{EC}$ at $0.5,1$ and $2 \mathrm{ml} /$ lit in combination with $P$. juliflora (1:5) showed highest inhibition zone of 22.67, 23.67 and $26.67 \mathrm{~mm}$ respectively. Followed by $P$. juliflora $(1: 5)+$ chlorpyrifos $20 \%$ EC with $22.00,22.33$ and $26.67 \mathrm{~mm}$ at $1.5,2$ and $3 \mathrm{ml} /$ lit respectively and $P$. juliflora $(1: 5)+$ dichlorovas 76\% EC (20.00, 22.33 and 25.33 $\mathrm{mm}$ at $0.25 \%, 0.5 \%$ and $1.0 \%$ respectively) and there was no inhibition of bacterial growth when insecticides tested alone at their three different concentrations.

Hulloli et al., (1998) recorded neem products can act as synergistically with antibiotics (Streptocyline and kanamycin)/fungicides (ridomylmancozeb) and reduced their hazardous effects. The MIC (minimal inhibitory concentration) of certain mixture was effective at lower concentrations than the MIC of individual chemicals indicating that neem formulations have synergistic activity when combined with antibiotics and fungicides. It is thus clear that addition of neem products to chemicals not only reduced their MIC but also eliminated the hazards of the development of the drug mutants.

Kumbhar et al., (2001) evaluated the combined formulation of Capsicum annuum (green chilli), Zingiber officinale and Allium sativum with 5 per cent cyclohexane exhibited better potential than individual formulation and crude extracts against $A$. niger, A. flavus and $F$. oxysporum under in vitro.

The data in Table 3, recorded that the efficiency of $G$. indica (1:5) in combination with different bactericides, contact and systemic fungicides and insecticides in three different concentrations under in vitro on the growth of $X$. axonopodis. pv. punicae. Among the pesticides tested, the bactericide 2-bromo2-nitropropane-1,3-diol (bronopol) in combination with $G$. indica (1:5) showed maximum inhibition zone of 27.33, 32.00 and
$34.00 \mathrm{~mm}$ in diameter at 250, 500 and 750 ppm concentration respectively. Streptocycline at 250, 500 and $750 \mathrm{ppm}$ in combination with $G$. indica (1:5) recorded with $25.33,28.00$ and $32.67 \mathrm{~mm}$ respectively. However, 2-bromo-2-nitropropane-1,3diol(bronopol) and Streptocycline alone recorded 24.67 and $22.00 \mathrm{~mm}$ at $250 \mathrm{ppm}$, 25.33 and $24.00 \mathrm{~mm}$ at $500 \mathrm{ppm}$ and 28.67 and $26.67 \mathrm{~mm}$ at $750 \mathrm{ppm}$ respectively.

In case of systemic fungicide tested along with $G$. indica $(1: 5)$ at their three different concentrations tested. Among them propiconazole $25 \% \mathrm{EC}$ at $0.5,1$ and $2 \mathrm{ml} / \mathrm{lit}$ along with $G$. indica (1:5) showed highest inhibition zone of 22.00, 24.00 and $26.00 \mathrm{~mm}$ respectively. The next best treatments were, G. indica $(1: 5)+$ hexaconazole $(20.00,23.33$ and $25.00 \mathrm{~mm}), \quad$ G. indica $(1: 5)+$ difenconazole (18.00, 20.33 and $22.67 \mathrm{~mm}$ ) and $G$. indica (1:5) + carbendazim (17.00, 20.00 and $21.00 \mathrm{~mm}$ ) at 1,2 and $3 \mathrm{~g} / \mathrm{lit}$ respectively. However the propiconazole $25 \%$ EC (11.33, 12.67 and $15.33 \mathrm{~mm})$, hexaconazole 5 EC (9.67, 12.00 and 14.33 $\mathrm{mm})$, difenconazole $(10.67,12.33$ and 14.67 $\mathrm{mm}$ ) recorded zone of inhibition in diameter at $0.5,1$ and $2 \mathrm{ml} /$ lit respectively. No inhibition zone at their three concentrations when tested alone. Whereas carbendazim 50wp alone recorded no inhibitory action. Results also revealed that all systemic fungicides are compatible with $G$. indica $(1: 5)$.

In case of contact fungicides all the three fungicides compatible with $G$. indica (1:5) at their three different concentrations tested. Among contact fungicides, copper oxychloride 50 WP at 1,2 and $3 \mathrm{~g} /$ lit along with $G$. indica (1:5) showed highest inhibition zone of 21.33, 24.67 and $27.00 \mathrm{~mm}$ respectively. Followed by $G$. indica $(1: 5)+$ mancozeb 75 WP (20.67, 23.00 and 26.33 $\mathrm{mm})$, G. indica $(1: 5)+$ chlorothalonil $75 \mathrm{WP}$ 
(19.00, 20.67 and $23.00 \mathrm{~mm})$ at 1,2 and 3 g/lit respectively. When the copper oxychloride 50 WP (14.67, 21.33 and 23.33 $\mathrm{mm})$, mancozeb $75 \mathrm{WP}$ (11.33, 15.67 and $24.00 \mathrm{~mm}$ ) and chlorothalonil $75 \mathrm{WP}(8.76$, 14.00 and $17.67 \mathrm{~mm}$ ) zone of inhibition in diameter at 1, 2 and $3 \mathrm{~g} /$ lit respectively, when they tested alone.

In case of insecticides all three are compatible with $G$. indica (1:5) at their three different concentrations tested. Among the insecticides dimethoate $30 \% \mathrm{EC}$ at $0.5,1$ and $2 \mathrm{ml} / \mathrm{lit}$ in combination with $G$. indica (1:5) showed highest inhibition zone of 19.67, 21.33 and $24.67 \mathrm{~mm}$ respectively. Followed by $G$. indica (1:5) + chlorpyrifos 20\% EC (18.67, 20.67 and $23.00 \mathrm{~mm}$ at $1.5,2$ and $3 \mathrm{ml} / \mathrm{lit}$ respectively) and $G$. indica (1:5) + dichlorovas $76 \%$ EC (17.67, 20.00 and 21.33 $\mathrm{mm}$ at $0.25 \%, 0.5 \%$ and $1.0 \%$ respectively) and there was no inhibition of bacterial growth when insecticides tested alone at their three different concentrations.

Meena et al., (2017) reported efficacy of antibiotic, fungicide and botanical were examined individually as well as in combinations for two consecutive years. Combined application of tetracycline @ 250 ppm + copper hydroxide @1000 ppm along with garlic clove extract @ 20 per cent effectively suppressed the disease and recorded the minimum of 16.20 PDI with 76.72 per cent reduction over control than the individual treatments. Application of garlic cloves extract along with tetracycline @ 250 ppm and copper hydroxide @1000 ppm had synergistic effect on the suppression of the bacterial pathogen associated with pomegranate. Similar response were also recorded during 2014 with the foliar application of tetracycline + copper hydroxide + garlic cloves extract, which had a minimum of 13.40 PDI with 79.51 per cent disease reduction over control. It was comparatively superior over the standard check namely, streptocycline + copper oxychloride. However, individual application of tetracycline@250 ppm or copper hydroxide @ 1000 ppm or, garlic cloves extract were least effective in the suppression of pomegranate bacterial blight. Therefore, it can be hypothesized that combine application of these chemicals with different mode of action have very good response in managing plant disease due to their synergistic effect. Besides, it also reduced the chance of multidrug resistance development within pathogen's population.

\section{References}

Bora, C. L. and Kataki, L., 2014, Xanthomonas axonopodis pv. punicaeA new threat to pomegranate plants in assam. Indian J. Hill Farm., 27(1): 100101.

Chand, R. and Kishun, R., 1991, Studies on bacterial blight (Xanthomonas campestris pv. punicae) of pomegranate. Indian Phytopathol. 44: 370-371.

Holder, I. A. and Boyse, S. T., 1994, Agar well diffusion assay testing of bacterial susceptibility to various antimicrobials in concentrations non-toxic for human cells in culture. Burns. 5: $426-429$.

Hulloli, S. S., Singh, R. P. and Verma, J. P., 1998, Management of bacterial blight of cotton induced by Xanthomnas axonopodis pv. malvacerum with the use of neem based formulations. Indian Phytopathol. 51(1): 21-25.

Kamble, K. G., 1990, Investigations on bacterial disease of pomegranate (Punica granatum). M. Sc. thesis, MAU Parbhani, Maharashtra (India).

Kanwar, Z.S., 1976, A note on bacterial disease of pomegranate (Punica granatum L.) in Haryana. Haryana $J$. Hort. Sci., 5: 177-180. 
Kumbhar, P.P., Kshama, M., Chawan Ujwala, B., Patil, Vidya, P., Nikubh Bendre, R.S. and Dewang, P.M., 2001, Antifungal and repellent potency of some spice extracts. Pestology, 25: 4446.

Meena, S. C., Chattopadhyay, A., Meena, M. K., Shah R., Rawal. P and Mali. B. L., 2017, Integration of chemicals and botanicals for the management of pomegranate bacterial blight caused by
Xanthomonas axonopodis pv. punicae. $J$ Mycol Pl Pathol, Vol. 4. No.1,

Rangaswamy, G., 1962, Pomegranate. In: Bacterial Plant Diseases in India. Asia Publication House, Bombay, p. 830.

Rani, U., Verma, K. S. and Sharma, K. K., 2001, Pathogenic potential of Xanthomonas axonopodis pv. Punicae and field response of different pomegranate cultivars. Plant Dis. Res., 16: $198-202$.

\section{How to cite this article:}

Jagadeesh Bagewadi, K.C. Kirankumar, R.K. Mestha and Chetankumar, K. 2018. In vitro Compatibility between Botanicals and Pesticides against Xanthomonas axonopodis pv. punicae. Int.J.Curr.Microbiol.App.Sci. 7(03): 1835-1842.

doi: https://doi.org/10.20546/ijcmas.2018.703.216 